\title{
Anti-reflective coating for multipatterning lithography
}

\author{
Douglas J. Guerrero, Steve Gibbons, Joyce Lowes, Ramil Mercado
}

Brewer Science, Inc., 2401 Brewer Drive, Rolla, MO 65401, USA

\begin{abstract}
New bottom anti-reflective coatings (BARCs) have been developed that can be incorporated into multiple patterning schemes utilizing scanner-track-only processes. The BARCs have modifiable optical properties and can be removed during the resist development step. Several dual patterning schemes were investigated for trench printing. The most promising process produced $110 \mathrm{~nm}$ trenches with approximately 1:1 space ratios. The etch characteristics of these BARCs under fluorinated and oxygenated gases were determined.
\end{abstract}

Keywords: BARC, wet developable, double patterning

\section{INTRODUCTION}

As features shrink even further for the 32-nm technology node and beyond, new materials and processing schemes are needed to achieve the target critical dimensions (CDs) for patterning. A method to achieve higher image density and tighter pitch without having to use more advanced imaging tools (shorter wavelength, higher NA) is to do multiple patterning steps. In this scheme, a pattern having large pitch is first printed. In a second step, new patterns are printed on the open spaces. This method allows for tighter pattern density while eliminating the need to print smaller structures. Several processes have been suggested in the literature ${ }^{1,2}$. The least favorable yet more straightforward process involves a lithography step followed by etching, followed by a second litho-etching series. This process is known as litho-etchlitho-etch (LELE), and, although proven ${ }^{3}$, it is becoming less attractive than other methods that utilize track-scanneronly processes. The additional etch steps are considered to be more time consuming and costly. The motivation of this research is to provide materials that can be used in processes carried out inside the scanner-track bay only, more specifically, organic materials for reflectivity control.

There have not been many reports on the subject of reflection control using organic films for multipatterning processes. Although commercially available organic bottom anti-reflective coatings (BARCs) can be utilized, the aggressive resist thickness being proposed $(<100 \mathrm{~nm})$ for the $32-\mathrm{nm}$ node requires a BARC that can be removed without an etch step. The ideal material would have to be removed during the resist development step and must have solvent resistance. A new anti-reflective coating with modifiable optical absorption ( $k$ value) has been developed which crosslinks and is insoluble in typical photoresist solvents. The coating is also imageable. The basic concept of using this material in a multipatterning process in several schemes will be explained.

\section{METHODOLOGY}

\subsection{General}

The BARC system consists of a polymer with absorbing unit attached to the backbone, multifunctional crosslinker, PAG, quencher, and solvent. The system is soluble in common industry solvents before BARC post-application bake (PAB), which produces a crosslinked film that is insoluble in resist solvent. However, after resist application, exposure, and post-exposure bake (PEB), the exposed area of BARC is soluble in developer. The chemistry has been described previously ${ }^{4-6}$. The films were coated at $160^{\circ} \mathrm{C}$ for 60 seconds to produce a $55-\mathrm{nm}$ film. The optical constants of the coated film were $n=1.67$ and $k=0.40$ as measured using a VASE ${ }^{\circledR}$ from J.A. Woollam Co., Inc.

Copyright 2008 Society of Photo-Optical Instrumentation Engineers. This paper was published in Proceedings of SPIE, Volume 6923, and is made available as an electronic reprint with permission of SPIE. One print or electronic copy may be made for personal use only. Systematic or multiple reproduction, distribution to multiple locations via electronic or other means, duplication of any material in this paper for a fee or for commercial purposes, or modification of the content of the paper are prohibited. 


\subsection{Lithography and etch}

Each BARC formulation was coated on a wafer on a TEL CLEAN TRACK ACT 8 and was in turn coated with an ArF commercial resist. Each coated wafer was exposed using an ArF scanner with a $0.85 \mathrm{NA}$, annular illumination with $0.93 / 0.62$ outer/inner sigma values. The resist thickness was $190 \mathrm{~nm}$. The resist underwent PAB and PEB (each $110^{\circ} \mathrm{C}$ for 60 seconds) and developed with 2.38\% TMAH developer for 45 seconds. The exposures used a dark field mask for trench printing. Blanket etch rates were determined in a Oxford Plasmalab 80+ system at 100 Watts and 50 mTorr of pressure using a mixture of $\mathrm{CF}_{4}, \mathrm{C}_{2} \mathrm{~F}_{6}$, Argon, and $\mathrm{O}_{2}$ gases for 45 second etch time.

\section{DATA AND RESULTS}

\subsection{Patterning processes}

Four patterning processes were studied and are illustrated in Figure 1. The goals of the process evaluation were to investigate the capabilities of a photo-decrosslinked BARC in multiple patterning schemes and to determine which process flow gave the best results using this type of material. The description and results for each process are described the sections below.

A

Resist Litho / Develop / Step / Resist Litho / Develop
B

Resist Litho / Step / Resist Liho / Develop
C

DBARC Litho / Develop Step / Resist Litho / Develop
D

Resist Litho/Develop/Strip Step / Resist Litho / Develop

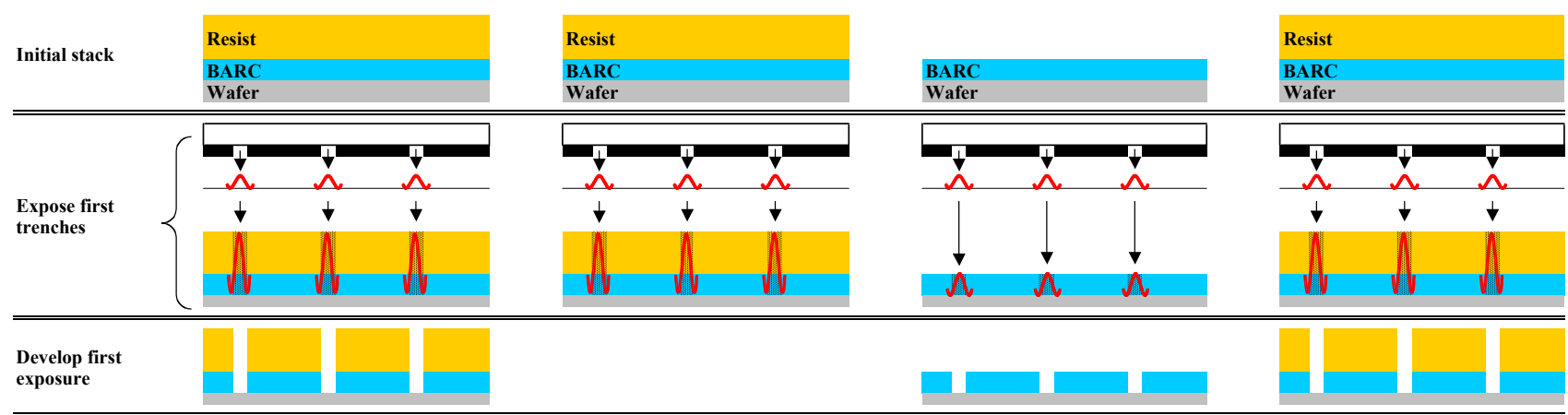

Strip the resist

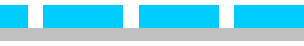

Coat with resist

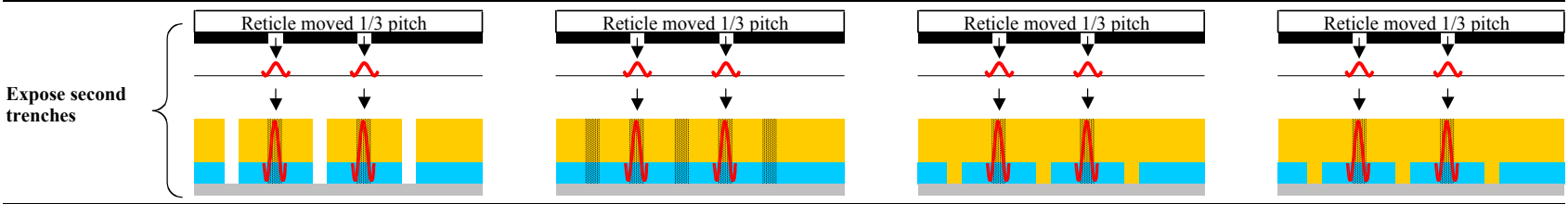

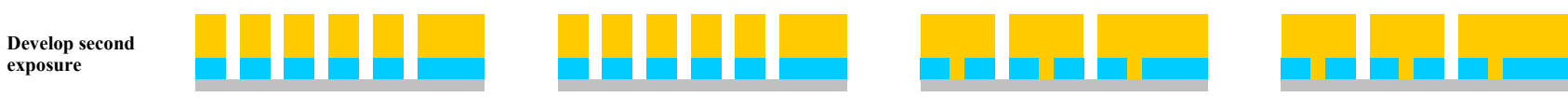

Figure 1. Multipatterning schemes used in evaluation of the BARC. 


\subsubsection{Litho-develop-litho-develop (Process A)}

In the first process (Figure 1A), the BARC and resist were coated, exposed, and developed. The wafer was reintroduced into the scanner and moved in the $\mathrm{x}$-axis by $1 / 3$ pitch distance and then exposed and developed once again. Figure 2 shows top-down CD-SEM photos of the trench array after the first and second photo steps. The results show that a higher density trench array could be printed.

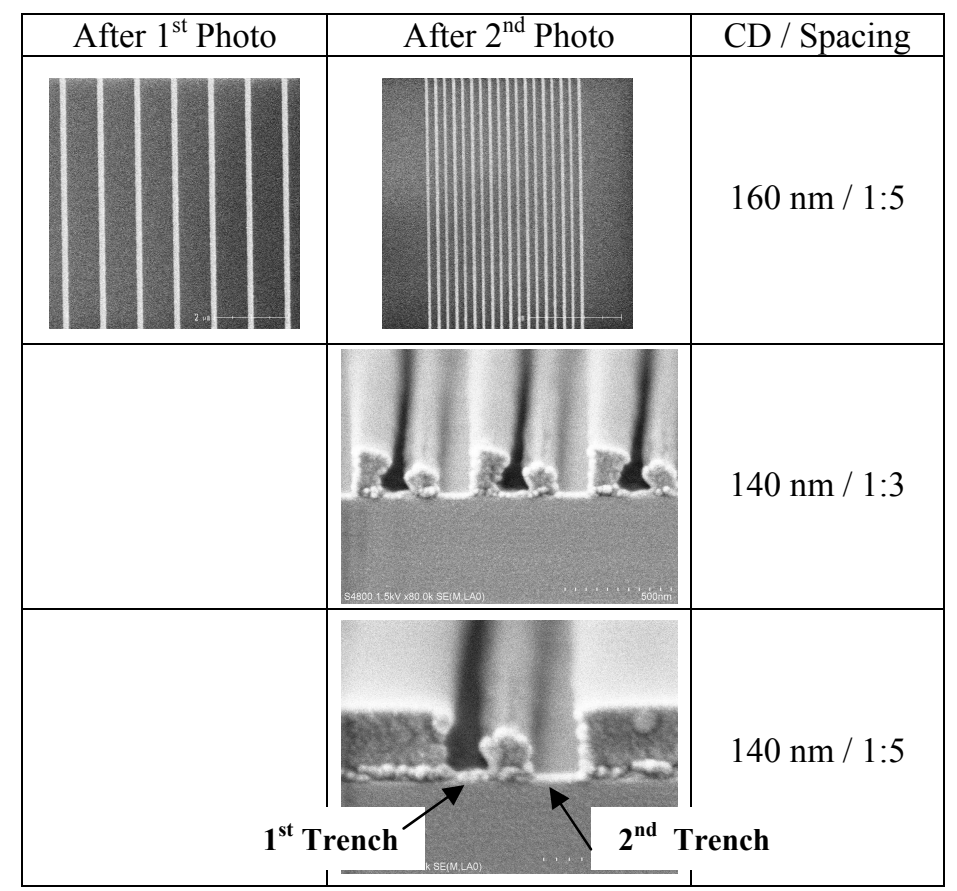

Figure 2. SEM photos of multiple patterning process using process A.

The main drawback to using this approach was that the first resist image underwent two develop steps. The double develop on the first image caused dark loss and deterioration of the first image (space between trenches). We were able to resolve $130-\mathrm{nm}$ trenches with some spacing collapse.

\subsubsection{Litho-litho-develop (Process B)}

In the second process (Figure 1B), the BARC and resist were coated and exposed. The develop step carried out in process A was skipped with the intention to eliminate dark loss. The wafer was then moved back into the scanner and translated in the $\mathrm{x}$-axis by $1 / 3$ pitch distance and then exposed and developed. After developing, the first and second images were created at once. The results show a high-density trench array as with process A but with less dark loss on the first image. A concern in this approach was the delay time between the first and second exposures. The delay time between exposures was $<1$ hour. However, both first and second trenches have the same CD as measured by CD-SEM. The space $\mathrm{CD}$ is not the same due to not having exact alignment between the first and second printing. In this experiment, no rigorous metrology was carried out to assess the effects of the delay time between exposures across the whole wafer. An advantage of process B over process A is that we were able to resolve smaller trenches $(110 \mathrm{~nm})$ without space collapse (Figure 3). 


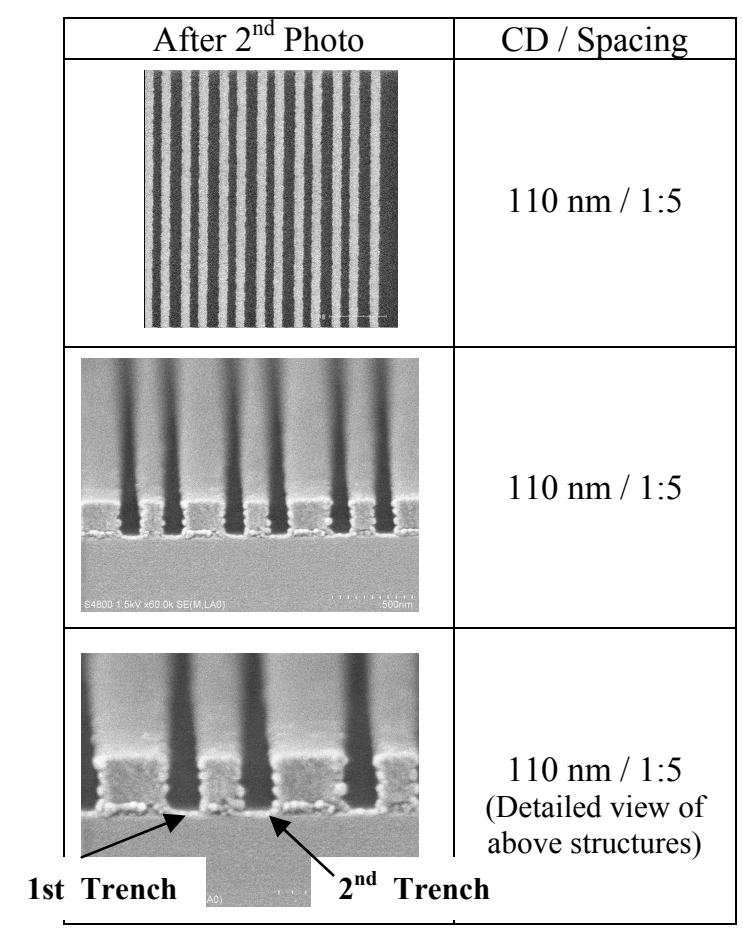

Figure 3. SEM photos of multipatterning process using process B.

\subsubsection{BARC-only litho-develop-litho-develop (Process C)}

In this process, illustrated in Figure 1C, the BARC was first imaged without a resist. Since the BARC is photosensitive, we wanted to explore the capabilities of performing the first imaging step without a resist. This process is also intended to eliminate dark loss due to double development (Process A) and CD changes that can occur due to exposure delay times (Process B). After the first exposure, the wafer is developed, coated with resist, and returned to the scanner. The wafer is then moved in the x-axis by $1 / 3$ pitch distance, exposed, and developed once again. Figure 4 below shows a cross-section SEM of the BARC-resist stack prior to removal of the resist. At the end of the process, the resist is stripped, giving a dense array. It is understood that a limitation would be the etch resistance of the BARC. The etch properties of these films are discussed in section 3.2 below.

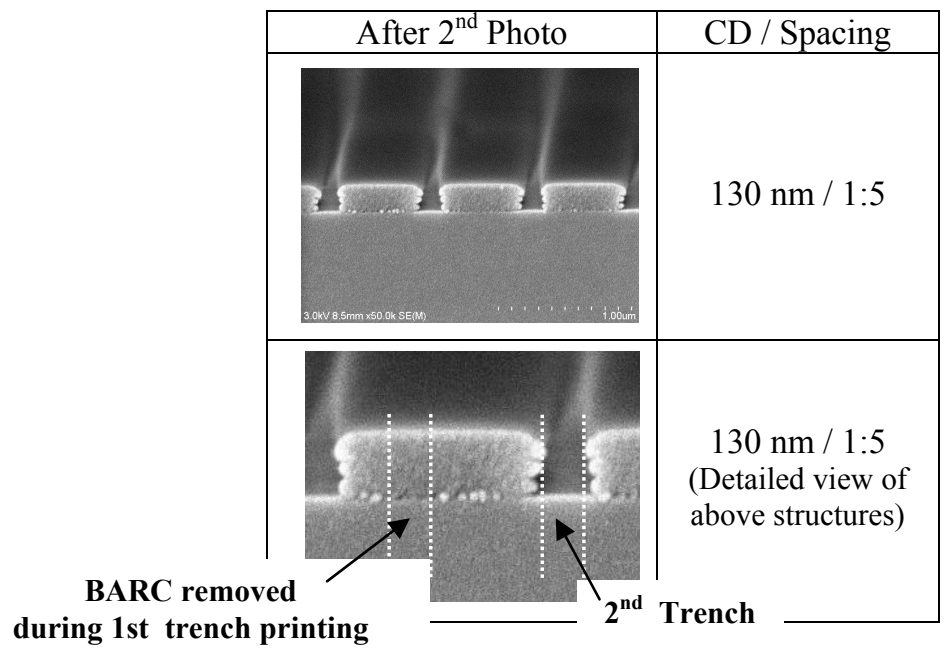

Figure 4. Cross-section SEM photo of BARC-resist prior to resist removal using process C. 
Process $\mathrm{C}$ main challenge was the ability to obtain good contrast prior to the second patterning step. The imaged BARC film is very thin compared to a conventional resist giving low contrast that caused overlay challenges. After resist strip, we did not obtain good quality SEM images of the dense trench array on the BARC.

\subsubsection{Litho-resist strip-litho-develop (Process D)}

The last process evaluated is very similar to the first process, except that the resist is stripped and recoated after the first develop step. This process is illustrated in Figure 1D. The goal of this process is also to eliminate dark loss, and maintain the resolution fidelity of the resist during both printing steps. In this process, the BARC and resist are coated and developed. The resist is then stripped and recoated before a second litho-develop pass. Because the BARC is crosslinked, it can be re-coated again with resist without stripping the first image printed on it. The results show that a high density trench array can be achieved.

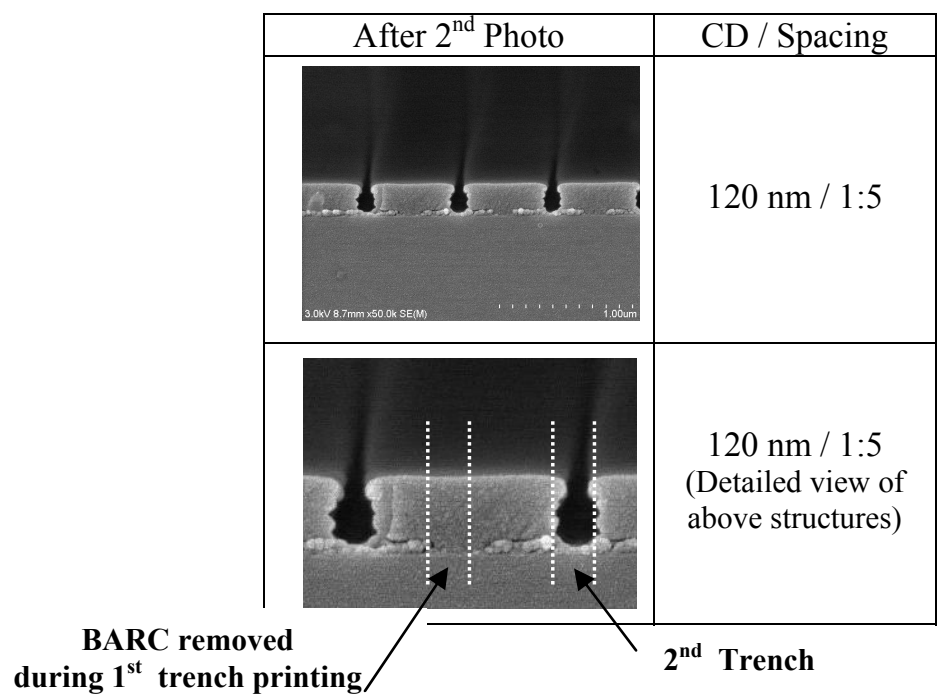

Figure 5. Cross-section SEM photo of BARC-resist prior to resist removal using process D.

\subsection{Etch characterization}

The blanket etch rates for the DBARC used in the multipatterning schemes were determined in a mixture of $\mathrm{CF}_{4}, \mathrm{C}_{2} \mathrm{~F}_{6}$, Argon and Oxygen to assess the merit of the patterned films as transfer layers. Table 1and 2 below show the conditions used. In order to obtain minimize end-point determination error that occurs when using thin films, thicker $270 \mathrm{~nm}$ films were used.

Table 1. Etch rates of DBARC in $\mathrm{CF}_{4}, \mathrm{Ar}$, and $\mathrm{O}_{2}$ mixtures.

\begin{tabular}{|c|c|c|c|c|}
\hline Run & CF4 (SCCM) & $\operatorname{Ar}(\mathrm{SCCM})$ & O2 (SCCM) & $\begin{array}{c}\text { Rate } \\
(\mathrm{nm} / \mathrm{min})\end{array}$ \\
\hline 1 & 50 & 0 & 0 & 77 \\
\hline 2 & 16.5 & 16.5 & 16.5 & 212 \\
\hline 3 & 0 & 25 & 25 & 245 \\
\hline 4 & 16.5 & 16.5 & 16.5 & 211 \\
\hline 5 & 0 & 0 & 50 & 260 \\
\hline 6 & 0 & 50 & 0 & 40 \\
\hline 7 & 33.5 & 8.5 & 8.5 & 159 \\
\hline 8 & 8.5 & 33.5 & 8.5 & 181 \\
\hline 9 & 25 & 0 & 25 & 237 \\
\hline 10 & 16.5 & 16.5 & 16.5 & 214 \\
\hline 11 & 25 & 25 & 0 & 69 \\
\hline 12 & 8.5 & 8.5 & 33.5 & 268 \\
\hline
\end{tabular}


Table 2. Etch rates of DBARC in $\mathrm{C}_{2} \mathrm{~F}_{6}, \mathrm{Ar}$, and $\mathrm{O}_{2}$ mixtures.

\begin{tabular}{|c|c|c|c|c|}
\hline Run & $\mathrm{C} 2 \mathrm{~F} 6(\mathrm{SCCM})$ & $\operatorname{Ar}(\mathrm{SCCM})$ & $\mathrm{O} 2(\mathrm{SCCM})$ & $\begin{array}{c}\text { Rate } \\
(\mathrm{nm} / \mathrm{min})\end{array}$ \\
\hline 1 & 50 & 0 & 0 & 59 \\
\hline 2 & 16.5 & 16.5 & 16.5 & 190 \\
\hline 3 & 0 & 25 & 25 & 259 \\
\hline 4 & 16.5 & 16.5 & 16.5 & 191 \\
\hline 5 & 0 & 0 & 50 & 273 \\
\hline 6 & 0 & 50 & 0 & 49 \\
\hline 7 & 33.5 & 8.5 & 8.5 & 115 \\
\hline 8 & 8.5 & 33.5 & 8.5 & 165 \\
\hline 9 & 25 & 0 & 25 & 196 \\
\hline 10 & 16.5 & 16.5 & 16.5 & 195 \\
\hline 11 & 25 & 25 & 0 & 63 \\
\hline 12 & 8.5 & 8.5 & 33.5 & 282 \\
\hline
\end{tabular}

The relationships between rate and gas mixture are shown in Figures 6a,b. In both halogenated gas mixtures, as the Oxygen content is reduced the etch rates decrease. The DBARC shows resistance to halogenated gases. The responses show that, within the experimental space, while the BARC might be an adequate for Poly-etch but it etches too fast for patterning carbon layers.
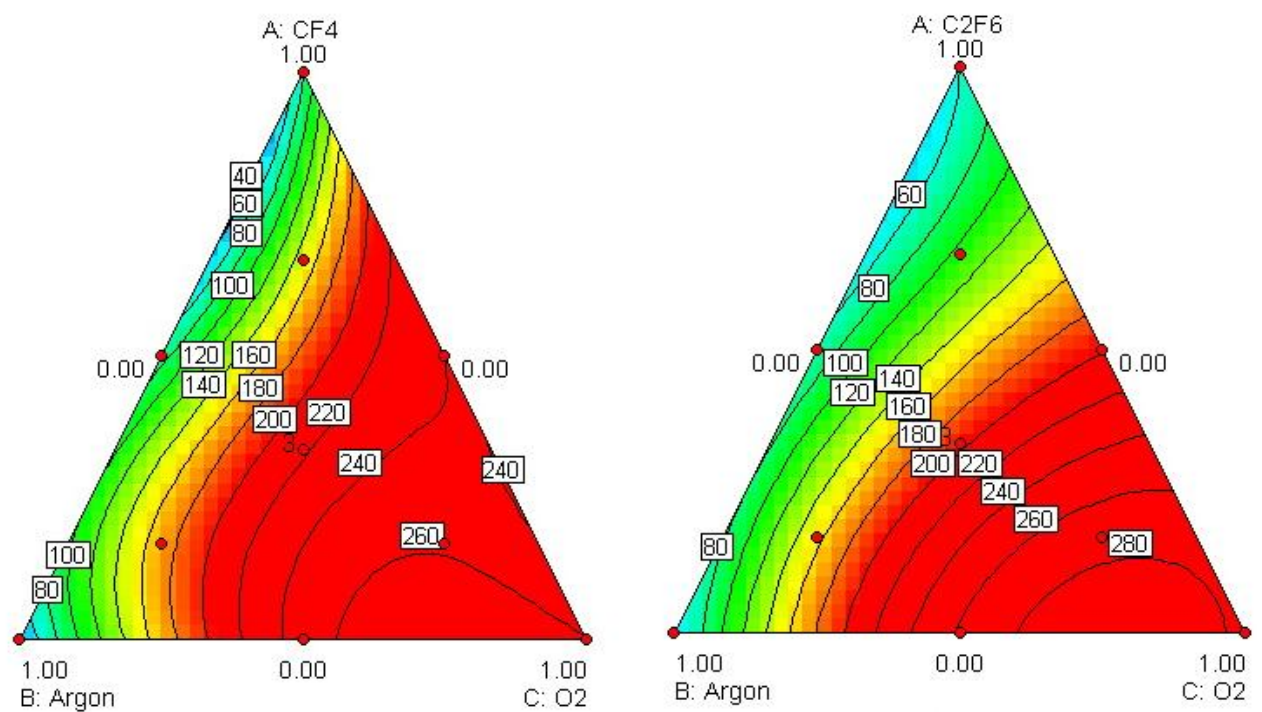

Figure 6. DBARC etch rates $(\mathrm{nm} / \mathrm{min})$ in a) $\mathrm{CF}_{4}$ and b) $\mathrm{C}_{2} \mathrm{~F}_{6}$ gas mixtures.

\section{CONCLUSIONS}

BARC coatings were developed for use in multipatterning lithography. Four processes were evaluated to assess the performance of the BARC in producing dense trench arrays. Process B gave the better results in spite of the concerns with exposure time delay between the two imaging steps. Trench sizes of $110 \mathrm{~nm}$ with approximately 1:1 space ratios were obtained. It was found that the BARC is capable of undergoing processes involving multiple coating and imaging steps. The etch rates in Oxygen rich gases needs further improvement to allow for pattern transfer to underlying substrates. Future work will focus in obtaining smaller CD's, pitch densities and etch resistance. 


\section{ACKNOWLEDGMENTS}

The authors would like to acknowledge the contributions of Nissan Chemical Industries for the use of their facility and equipment to perform the double patterning experiments. Additionally, we would like to thank Dr. Sangwoong Yoon and Takahiro Hamada for assisting in the conceptualization of the double patterning methodologies and for programming and training on the ArF scanner, respectively. The contribution for the etch work by Dr. Hao Xu and Charlyn Stroud of Brewer Science are gratefully acknowledged.

\section{REFERENCES}

1. M. Maenhoudt, J. Versluijs, H. Struyf, J. van Olmen, M. van Hove, "Double patterning scheme for sub-0.25 k1 single damascene structures at NA=0.75, $\lambda=193 \mathrm{~nm} "$, Proc. SPIE, 5754, 1508-1518, 2005.

2. Hung Jen Liu, Wei Hsien Hsieh, Chang Ho Yeh, Jan Shiun Wu, Hung Wei Chan, Wen Bin Wu, Feng Yi Chen, Tse Yao Huang, Chiang Lin Shih, Jeng Ping Lin, "Double patterning with multilayer hard mask shrinkage for sub-0.25 k1 lithography," Proc. SPIE, 6520, 65202J-1 - 65202J-8, 2007.

3. M. Dusa, J. Quaedackers, O.F.A. Larsen, J. Meessen, E. van der Heijden, G. Dicker, O. Wismans, P. de Haas, K. van Ingen Schenau, J. Finders, B. Vleeming, G. Storms, P. Jaenen, S. Cheng, M. Maenhoudt, "Pitch Doubling Through Dual Patterning Lithography Challenges in Integration and Litho Budgets." Proc. SPIE, 6520, 2007.

4. Douglas J. Guerrero, Ramil Mercado, Carlton Washburn, and Jim Meador, "Photochemical Studies on Bottom AntiReflective Coatings," J. Photopolym. Sci. and Technol., 19, No. 3, pp. 343-347, 2006.

5. Carlton Washburn, Alice Guerrero, Ramil Mercado, Douglas Guerrero, and Jim Meador, "Process development for developer-soluble bottom anti-reflective coatings (BARCs)," INTERFACE 2006: Proceedings of the $43^{\text {rd }}$ Microlithography Symposium, October 29-31, 2006.

6. Carlton Washburn, Ramil Mercado, Douglas Guerrero, Jim Meador, "Controlling CD and process window limits for implant patterning," Solid State Technology, 49, No. 10, pp. 53-56, 2006. 